(IQR 24-30) $(\mathrm{p}<0.001)$. Participants were able to identify and discuss a large number of theoretical and practical considerations in RTC ideation, design, conduct, interpretation and publication, and reached meaningful insights into generalizability of results, protocol violations, missing data, standardization of measurement instruments, concomitant medications, blinding, GCP, study monitoring, and others.

All trial participants enjoyed the simulation, and most (there were a few protocol violations) happily sipped their cup of coffee, with no adverse event. None was lost to follow up.

Conclusions Short-term learning goals were achieved in a rapid and entertaining way. Long-term utility of the teaching exercise will be measured by means of the number of future publications by the participants in high impact journals. The effects of coffee on cardiovascular outcomes will need a much larger sample size to be ascertained, but the effect on students' mood was striking, with no safety concerns.

\section{INTERNATIONAL PEER REVIEW AND DECISION-MAKING PRACTICES, BIAS, AND BURDEN: THE FUNDERS' PERSPECTIVE}

Katie Meadmore, Alex Recio-Saucedo, Kathryn Fackrell, Abby Bull, Amanda Blatch-Jones. National Institute for Health Research Evaluation, Trials and Studies Coordinating Centre (NETSCC), Southampton, UK

\subsection{6/bmjebm-2019-EBMLive.19}

Objectives Health research funding organisations are responsible for allocating funds for research in a fair, transparent and efficient way. Peer review, and often external peer review, is considered vital for the decision-making process. However, it is well established that peer review can be biased and burdensome for the applicant, peer reviewer and funding organisation. There is limited evidence to suggest which approaches have been considered by funding organisations and whether they have been evaluated to determine their applicability and generalisability. The purpose of this work is to contribute to the evidence gap around the value of alternative and/or innovative approaches to decision-making for grant fund allocation from the perspective of funders. The aim of this study was to identify and explore decision-making practices used by UK and international funding organisations for the allocation of funding for health-related research.

Method An online survey was distributed to UK and international health and health-related funding organisations (March/ April 2019). The survey collected information about current, past and future approaches to decision-making for grant fund allocation. The survey was sent to 63 funders (targeted list collated by National Institute of Health Research [NIHR]). Social media coverage was used to attract additional funding organisations. The survey focused at the level of a research programme rather than the overall organisation. Therefore, an entry could be provided for every research programme in an organisation. The survey had 3 sections: ${ }^{1}$ characteristics of the funding organisation; ${ }^{2}$ decision-making approaches currently being used and those used in the past; and $^{3}$ approaches that may be considered in the future. Participants were encouraged to provide additional information on what worked well, any potential drawbacks and suggestions for improvements. The survey was active for six weeks (ending 17 April 2019).

Results Preliminary descriptive statistics and thematic analysis were conducted on the data available at 3 weeks. Data was quality checked before analysis. From the initial analysis, 12 funding organisations responded to the survey providing coverage from charities and research councils in the health sector. The preliminary data shows that funders use triage, external peer review and face-to-face committee meetings. None reported use of open peer review, partial randomisation or sandpits. Key reported benefits of current systems included transparency and fairness, increased quality and relevance of applications, expert opinion, reduced burden through efficient processes, and consistency in assessment. The main drawbacks of current systems included the potential for bias, time and cost burden, and difficulty in securing external peer reviewers. The full analysis and results will be presented along with the recommendations from the study and its contribution to the wider portfolio of work under the NIHR Research on Research in-house programme.

Conclusions In the current research environment, funders have a responsibility to demonstrate fair, transparent and efficient decision-making practices, reducing bias and burden to the funder, peer reviewers and the applicant. There is a lack of understanding from the funder's perspective around what type of approaches work (or not) due to the lack of empirical evidence. This study aims to contribute to and further build on our understanding to provide robust evidence to enhance the decision-making process of grant allocation. Early findings indicate that funders are keen to explore alternative approaches to decision-making that facilitate the process (through reducing bias, time, monetary cost) whilst maintaining transparency, fairness and quality. The findings from this study will be important in the context of determining which approaches may be applicable and generalisable to use within organisations that fund health research.

\section{2 'WALKING THE TALK' A REAL-WORLD EXPERIENCE DEVELOPING A CLINICAL PRACTICE GUIDELINE FOR BAHRAIN}

1,2 Julie Sprakel, ${ }^{2}$ Helio Carrara, ${ }^{3}$ Brian Alper, ${ }^{4}$ Zbys Fedorowicz. ${ }^{1}$ Think Pink: Bahrain Breast Cancer Society, Manama, Bahrain; ${ }^{2}$ Gynecology and Obstetrics Department - Ribeirão Preto Medical School - University of São Paulo, Ribeirão Preto, Brazil, ${ }^{3} E B S C O$ Health, Boston, USA; ${ }^{4}$ Veritas Health Sciences Consultancy, London, UK

\subsection{6/bmjebm-2019-EBMLive.20}

Objectives To provide a high-quality, up-to-date, evidencebased resource with recommendations based on the GRADE approach and clinical algorithms to enable shared clinical decisions. Building on existing clinical practice guidelines (Bahrain, 2010) the completed work provides a local voice via the strategic championship of a mostly local multidisciplinary teamwhich includes consumer advocates and patients. The overall effort changed the perception of a role of a Non-Governmental Organisation (NGO) and highlighted a governmental/nongovernmental partnership could fill the gaps and better support the community. This comprehensive and contemporary resource can be used across the continuum of breast cancer care for both healthcare providers and patients, to better navigate the clinical pathways and provide best practice recommendations for informed and shared decision making.

Method The RAPADAPTE method provided rapid adaptation of guidelines and evidence resources and minimized unnecessary repetition, rather than developing the guideline de novo. RAPADAPTE builds on the well-established ADAPTE method and had been used to develop a similar breast cancer 